\title{
DOAÇÃO DE ÓRGÃOS: OPINIÃO E ENTENDIMENTO SOBRE MORTE ENCEFÁLICA DE ESTUDANTES UNIVERSITÁRIOS
}

\author{
Organ Donation: opinion and knowledge of college students about brain death
}

Mateus Germano Scaglioni Tessmer1, Grégore Iven Mielke², Franklin Correa Barcellos ${ }^{3}$,Bruna Pinheiro de Moraes4, Chiara Scaglioni Tessmer Gatto ${ }^{5}$

\begin{abstract}
RESUMO
Objetivo: Identificar a prevalência de intenção de doar órgãos em uma amostra de estudantes universitários, definir quais são os principais motivos para não doação de órgãos e verificar o entendimento e aceitação sobre morte encefálica. Métodos: A amostra foi composta por 485 alunos ingressantes no ano de 2008. Avaliou-se a opinião dos estudantes sobre doação de órgãos utilizando perguntas sobre a intenção de serem doadores, os motivos para não doar e, também, duas perguntas iguais, com exceção da substituição da palavra morte por "morte encefálica". Resultados: Dos indivíduos entrevistados 42,3\% tinham menos de 20 anos. Sobre doação de órgãos $65,1 \%$ (n=311) dos estudantes responderam que tinham a intenção de doar seus órgãos após a morte. Entretanto, 40,2\% (n=132) afirmaram não ter informado a qualquer parente sua intenção de doar. Segundo os estudantes, "desconhecimento do tema" é o principal o motivo para a não doação de órgãos postmortem. A maioria dos respondentes $(87,1 \%)$ autorizaria a doação dos órgãos de seu parente morto, se ele tivesse previamente declarado esse desejo. Entretanto, somente 60,3\% autorizariam a doação de órgão de um familiar se o mesmo estivesse em morte encefálica. Caso o entrevistado desconhecesse a vontade do familiar sobre a doação de órgão somente $34 \%$ dos entrevistados autorizaria a doação. Conclusão: Quando foi utilizado o termo morte encefálica ao invés de morte a taxa de intenção de doação de órgão diminuiu $26 \%$ demonstrando a não aceitação por parte dos entrevistados do termo morte encefálica como sinônimo de morte. Assim, julgamos que um maior entendimento e aceitação sobre morte encefálica por parte dos universitários é necessário, tornando-os exemplos a serem seguidos pela população, medida esta que pode ser alcançada com introdução de conceitos básicos desse assunto em todos os cursos de graduação.
\end{abstract}

Descritores: Doação de órgão, Transplante, Transplante de Órgãos, Doadores de Órgãos, Estudantes, Conhecimento, Atitude

\footnotetext{
Instituições:

1 Acadêmico da Faculdade de Medicina da Universidade Federal de Pelotas. Pelotas, RS, Brasil.

2 Programa de Pós-graduação em Epidemiologia, Universidade Federal de Pelotas, RS, Brasil.

${ }^{3}$ Programa de Pós-graduação em Epidemiologia, Universidade Federal de Pelotas e Centro de Ciências da Saúde e da Vida da Universidade Católica de Pelotas. Pelotas, RS, Brasil

${ }^{4}$ Acadêmica da Faculdade de Medicina da Universidade Católica de Pelotas. Pelotas, RS, Brasil.

${ }^{5}$ Instituto do Coração do Hospital das Clínicas da Faculdade de Medicina da Universidade de São Paulo, São Paulo, SP, Brasil
}

\section{Correspondência:}

Mateus Germano Scaglioni Tessmer

Av. São Francisco de Paula, 2592, Areal - CEP 96055-630 - Pelotas - RS

Tel: (53) 3228-4199

e-mail: mateustessmer@hotmail.com

\section{INTRODUÇÂO}

Nas duas últimas décadas, devido à grande melhora de sobrevida e qualidade de vida dos transplantados de órgãos, as indicações de transplantes e o número de pacientes cresceram significativamente., ${ }^{1,2}$ Isso resultou em um aumento de até $70 \%$, durante a última década, no número de pacientes na lista de espera. ${ }^{3}$ Entretanto, a disponibilidade de órgãos é muito menor do que a demanda para transplantes.

O Conselho Federal de Medicina (CFM) publicou em 1997 as normas para diagnóstico de ME. Os critérios clínicos devem ser registrados no termo de declaração de ME por dois médicos experientes, não necessariamente neurologistas, em intervalo que varia conforme a faixa etária de cada paciente. Ressalta-se a obrigatoriedade de realizar exames complementares demonstrando completa ausência de função cerebral. ${ }^{4}$ Portanto, a morte encefálica, sob o ponto de vista médico e ético, equivale à morte de um indivíduo, já que o cérebro é fundamental para a função integrativa do organismo. ${ }^{5}$

Através de dados publicados pelo Registro Brasileiro de Transplantes (RBT), veiculado à ABTO (Associação Brasileira de Transplante de Órgãos), sabe-se que no primeiro semestre de 2011 a taxa de doadores efetivos, em relação ao ano de 2010 , aumentou em $6,7 \%$, passando de 9,6 para 10,3 doadores por milhão de habitantes (pmp). Porém, apesar do número de doadores estar aumentando progressivamente, chegando próximo à meta estipulada para este ano, 11,5 pmp, percebe-se que a quantidade de transplantes realizados ainda permanece muito abaixo da 
necessidade, possivelmente devido à persistente alta taxa de negativa familiar, que foi de $52 \%$, além de outros motivos, como à falta de infraestrutura adequada. ${ }^{6}$

Pesquisas apontam que a desinformação sobre temas básicos desse método terapêutico prejudica a doação de órgãos e a maior abrangência do transplante como tratamento. ${ }^{7}$ Existem na literatura evidências demonstrando que estudantes do ensino médio e superior apresentaram significativo índice de desconhecimento sobre o assunto ME. ${ }^{8-10}$ Também, segundo uma pesquisa realizada na cidade de Campinas-SP, constatou-se que não só o altruísmo, mas também uma maior escolaridade e conhecimento sobre doação de órgãos dos indivíduos foram importantes marcadores favoráveis à doação. ${ }^{11}$

Assim sendo, este trabalho teve por objetivos identificar a prevalência da intenção de doar órgãos em uma amostra de estudantes universitários, comprovar, por meio de um método científico, quais são os motivos mais determinantes na recusa ou aceitação para os jovens tornarem-se doadores e verificar o entendimento e a aceitação do termo morte encefálica.

\section{MÉTODOS}

Foi realizado um estudo transversal no primeiro semestre de 2008, com alunos do primeiro ano da Universidade Federal de Pelotas (UFPel). Para selecionar a amostra, realizou-se um levantamento do número de vagas para o vestibular de 2008 , de acordo com as informações disponibilizadas pelo Departamento de Registros Acadêmicos (DRA-UFPEL), conforme as cinco áreas dos cursos de graduação: Ciências Agrárias, Ciências Biológicas, Ciências Exatas, Ciências Humanas e os cursos do Instituto de Letras e Artes. De forma a garantir a representatividade da amostra, o processo amostral foi realizado em múltiplos estágios:

Identificação da proporção de alunos ingressantes segundo a área acadêmica;

Sorteio aleatório dos cursos dentro de cada área, de forma a obter o número de alunos estimado, respeitando a proporcionalidade de cada área;

Seleção dos alunos matriculados no primeiro semestre dos cursos sorteados, que estavam presentes em sala de aula no momento da visita dos entrevistadores.

Para a coleta de dados foram utilizados questionários auto aplicados em sala de aula, os quais eram compostos por questões gerais sobre aspectos de saúde. Os questionários eram anônimos e os participantes assinaram um termo de consentimento livre e esclarecido. A fim de testar os instrumentos e a metodologia proposta para coleta de dados, inicialmente foi realizado um estudo piloto no qual foram aplicados questionários em alunos da Escola Superior de Educação Física da UFPel que não haviam sido selecionados para o estudo.

Para o estudo foram coletadas as seguintes variáveis sociodemográficas: sexo (masculino e feminino), idade $(<20$ anos; 20 a 24 anos e $\geq 25$ anos), tipo de escola frequentada no ensino médio (pública ou particular), local de moradia (com pais ou parentes, em pensionato ou república, em casa dividida com amigos ou sozinho) e nível socioeconômico (A, B, C, D/E). O nível socioeconômico foi avaliado por meio do Critério de Classificação Econômica Brasil, baseado no levantamento de bens de consumo, escolaridade do chefe da família e presença de empregada doméstica.

A avaliação a respeito do entendimento sobre morte encefálica, a intenção de autorizar a doação de órgãos de familiar após sua morte, quando este havia manifestado sua vontade de ser doador e também quando o familiar não manifestou a sua intenção, deu-se através das seguintes questões:

1) "Imagine que um parente seu tivesse lhe avisado sobre a vontade de ser doador de órgãos. O médico lhe avisou que esse seu parente morreu. Você autorizaria a doação de órgãos desta pessoa?”;

2) "Imagine que um parente próximo não tenha discutido com você sobre o tema doação de órgãos. O médico lhe avisou que esse parente está com morte cerebral. Você autorizaria a doação?";

3) "Você tem a intenção de doar algum órgão do seu corpo?";

4) "Você já avisou algum parente próximo sobre sua intenção de doar seus órgãos?";

O entendimento de morte encefálica foi verificado por meio de realização das questões 1 e 2 (citadas anteriormente), apenas com a substituição da palavra "morte" por "morte encefálica", sendo analisada a diferença entre as respostas afirmativas com as duas abordagem. Metodologia semelhante a esta já foi utilizada por outros autores. ${ }^{12,13}$

Foram considerados com intenção de doar órgãos os indivíduos que responderam afirmativamente à questão 4 (tabela 1).

Tabela 1: Classificação dos universitários de acordo com a intenção de doarem seus órgãos e de seus parentes.

\begin{tabular}{|c|c|c|c|}
\hline $\begin{array}{l}\text { QUESTÕES SOBRE DOAÇÃO } \\
\text { DE ÓRGÃOS }\end{array}$ & $\begin{array}{l}\text { SIM } \\
\mathrm{N}(\%)\end{array}$ & $\begin{array}{l}\mathrm{NÃO} \\
\mathrm{N}(\%)\end{array}$ & $\begin{array}{l}\text { INDECISOS } \\
\mathrm{N}(\%)\end{array}$ \\
\hline $\begin{array}{l}\text { Imagine que um parente seu } \\
\text { tivesse avisado sobre a vontade } \\
\text { de ser doador de órgãos. O } \\
\text { médico lhe avisou que esse } \\
\text { seu parente morreu. Você } \\
\text { autorizaria a doação de órgãos } \\
\text { desta pessoa? ( } n=482)\end{array}$ & $\begin{array}{c}420 \\
(87,1)\end{array}$ & $\begin{array}{c}13 \\
(2,7)\end{array}$ & $\begin{array}{c}49 \\
(10,2)\end{array}$ \\
\hline $\begin{array}{l}\text { Imagine que outro parente } \\
\text { próximo seu tivesse avisado } \\
\text { sobre sua vontade de ser } \\
\text { doador de órgãos. O médico lhe } \\
\text { avisou que esse seu parente } \\
\text { está com morte cerebral. Você } \\
\text { autorizaria a doação de órgãos } \\
\text { desta pessoa? }(n=479)\end{array}$ & $\begin{array}{c}289 \\
(60,3)\end{array}$ & $\begin{array}{c}65 \\
(13,6)\end{array}$ & $\begin{array}{c}125 \\
(26,1)\end{array}$ \\
\hline $\begin{array}{l}\text { Imagine que um parente } \\
\text { próximo não tenha discutido } \\
\text { com você sobre o tema doação } \\
\text { de órgãos. O médico lhe avisou } \\
\text { que esse parente está com } \\
\text { morte cerebral. Você autorizaria } \\
\text { a doação? }(n=477)\end{array}$ & $\begin{array}{c}165 \\
(34,6)\end{array}$ & $\begin{array}{c}155 \\
(32,5)\end{array}$ & $\begin{array}{c}157 \\
(32,9)\end{array}$ \\
\hline $\begin{array}{l}\text { Você tem a intenção de doar algum } \\
\text { órgão do seu corpo? }(n=478)\end{array}$ & $\begin{array}{c}311 \\
(65,1)\end{array}$ & $\begin{array}{c}50 \\
(10,5)\end{array}$ & $\begin{array}{c}117 \\
(24,5)\end{array}$ \\
\hline $\begin{array}{l}\text { Você já avisou algum parente } \\
\text { próximo sobre sua intenção de } \\
\text { doar seus órgãos? }(n=328)\end{array}$ & $\begin{array}{c}196 \\
(59.8)\end{array}$ & $\begin{array}{c}132 \\
(40.2)\end{array}$ & - \\
\hline
\end{tabular}


Os dados foram todos duplamente digitados no programa Epi-Info, versão 6.0. As análises dos dados foram realizadas no programa estatístico Stata, versão 10, sendo realizadas análises descritivas com distribuição de frequências entre grupos. Os resultados foram considerados estatisticamente significantes para um valor $\mathrm{p}<0,05$.

O estudo foi submetido e aprovado pelo Comitê de Ética em Pesquisa da Escola Superior de Educação Física da Universidade Federal de Pelotas recebendo aprovação sob protocolo n ${ }^{\circ}$ 022/2008.

\section{RESULTADOS}

Foram selecionados 16 cursos representando as cinco áreas acadêmicas. No total, 485 alunos responderam ao questionário.

A tabela 2 apresenta descrição de algumas características socioeconômicas, demográficas e biológicas da amostra. A média de idade dos universitários foi de 22,6 anos (DP 7,3) [IC95\% 21,9 -23,2], sendo a idade mínima 17 e máxima 67 anos. A maioria dos estudantes era de cor branca $(87,8 \%)$, do sexo feminino $(53,9 \%)$, moravam com os pais ou parentes $(66,8 \%)$, eram solteiros $(85,0 \%)$, foram classificados com nível sócio econômico da categoria $\mathrm{B}$ $(49,2 \%)$ e realizaram o ensino médio em escola pública $(74,7 \%)$.

Tabela 2: Descrição da amostra segundo variáveis demográficas e socioeconômicas, Universidade Federal de Pelotas 2008.

\begin{tabular}{|c|c|c|}
\hline Variáveis & $\mathrm{N}^{\mathrm{ab}}$ & $\%$ \\
\hline \multicolumn{3}{|l|}{ Sexo } \\
\hline Masculino & 220 & 46,1 \\
\hline Feminino & 257 & 53,9 \\
\hline \multicolumn{3}{|l|}{ Cor da pele } \\
\hline Branca & 424 & 87,8 \\
\hline Não branca & 59 & 12,2 \\
\hline \multicolumn{3}{|l|}{ Idade (anos) } \\
\hline$<20$ & 204 & 42,3 \\
\hline 20 a 24 & 183 & 38,0 \\
\hline$>25$ & 95 & 19,7 \\
\hline \multicolumn{3}{|l|}{ Nível socioeconômico } \\
\hline A & 73 & 15,8 \\
\hline B & 227 & 49,2 \\
\hline$C / D$ & 161 & 34,9 \\
\hline \multicolumn{3}{|l|}{ Tipo de escola (ensino médio) } \\
\hline Pública & 358 & 74,7 \\
\hline Particular & 112 & 23,4 \\
\hline Parcialmente em escola particular & 6 & 1,3 \\
\hline Outra & 3 & 0,6 \\
\hline \multicolumn{3}{|l|}{ Local de moradia } \\
\hline Pais ou parentes & 320 & 66,8 \\
\hline Pensionato, república & 20 & 4,2 \\
\hline Casa dividida com amigos & 62 & 12,9 \\
\hline Sozinho & 33 & 6,9 \\
\hline Outro & 44 & 9,1 \\
\hline
\end{tabular}

a Tamanho da amostra igual a 485 indivíduos;

b Numero máximo de informações perdidas igual a 24 para a variável nível socioeconômico.
Quando questionado aos alunos se eles tinham a intenção de doar seus órgãos após a morte, $65,06 \%(\mathrm{n}=311)$ responderam que sim. Destes, 40,24\% (n=132) afirmaram não ter informado a qualquer parente sua intenção de doar. Sendo que $31,40 \%(n=103)$ já informaram a dois ou mais parentes próximos, $17,38 \%(\mathrm{n}=57)$ informaram a sua mãe e 5,49\% $(\mathrm{n}=18)$ manifestaram ao marido, esposa ou companheiro sua vontade de ser doador de órgãos. No que se refere à autorização da doação de órgãos de familiares, a maioria, 87,14\% $(n=420)$, autorizaria a doação após a morte do familiar quando se sabia a intenção do familiar quanto à doação de órgãos. Entretanto, quando foi utilizada a expressão "morte encefálica" significando "morte", dentre os alunos que responderam positivamente à questão anterior, $11,69 \%(n=11)$ não autorizariam a doação mesmo conhecendo a vontade do familiar, $21,43 \%(n=90)$ mostraram-se indecisos e $0,48 \%(n=2)$ não responderam $(\mathrm{p}<0,001)$. Dessa forma, no momento em que se utilizou o termo "morte encefálica" como sinônimo de morte, apenas 60,33\% $(n=289)$ dos entrevistados manifestaram a intenção de autorizar a doação quando conheciam a vontade do parente. Quando o tema sobre intenção de doar não havia sido discutido previamente entre o entrevistado e seu parente, apenas um terço dos respondentes $34,59 \%(n=165)$ autorizaria a doação quando o familiar estivesse com diagnóstico de morte encefálica (tabela 1).

Quando foi questionado quais seriam os motivos para que as pessoas não doassem seus órgãos após a morte, 28,31\% responderam "desconhecimento do tema", 23,29\% responderam "não quer ter o corpo mutilado", 18,95\% responderam "medo de não estar morto" e $9,59 \%$ responderam "não acredita no sistema de saúde (médicos)" (tabela 3).

Tabela 3: Opinião dos universitários sobre o que pode levar as pessoas a não doar seus órgãos após sua morte.

\begin{tabular}{lll}
\hline Motivo & $\mathrm{N}$ & $\%$ \\
\hline Desconhecimentos do tema & 124 & 28,3 \\
Não quer ter o corpo mutilado & 102 & 23,29 \\
Medo de não estar morto & 83 & 18,95 \\
Não acredita no sistema de saúde (médicos) & 42 & 9,59 \\
Egoísmo & 38 & 8,68 \\
Religião & 35 & 7,99 \\
Outros & 14 & 3,20 \\
\hline
\end{tabular}

\section{DISCUSSÃO}

O presente estudo analisa o entendimento e a aceitação dos estudantes em relação à morte encefálica como critério de morte. Foi analisada essa população especificamente por representarem formadores e multiplicadores da opinião pública, podendo atuar positivamente no esclarecimento das dúvidas da população sobre o diagnóstico de morte encefálica e o processo da doação de órgãos.

Devido à falta de órgãos, a fila de espera e o número de pacientes que morrem enquanto aguardam o transplante em vários países, inclusive nos Estados Unidos, Europa e Brasil, são expressivos. ${ }^{14}$ Já, a incidência de morte encefálica em unidades de terapia intensiva de adultos na Europa e EUA situa-se ao redor de $12 \%$, 
sendo semelhante à descrita em trabalhos realizados no Brasil e EUA envolvendo unidades de tratamento intensivo pediátrico. ${ }^{16,17}$ Entretanto, a grande maioria desses pacientes não se torna doador, como demonstrou o estudo retrospectivo, realizado pelo Núcleo de Estudos em Ética em Pediatria-Brasil, no ano de 2007,13 o qual verificou que somente $9,8 \%$ dos diagnosticados com morte encefálica tornaram-se doadores efetivamente. Entre as possíveis causas para essa discrepância estão a não aceitação dos familiares, a falta de engajamento das equipes médicas e a falta de integração entre as UTIs e os centros de transplantes. Numa revisão de literatura realizada no ano de 2010, encontrou-se que o um dos fatores que mais influenciou na recusa dos familiares para a doação foi o não entendimento da ME. ${ }^{18}$

Em 2007, foi realizado um estudo retrospectivo no estado de Sergipe, o qual identificou a taxa de $9,22 \%$ entre possíveis doadores dos 1388 laudos necroscópicos analisados daquele ano, sendo que a faixa etária predominante foi de 21 a 40 anos, com 54,4\% dos indivíduos. ${ }^{19}$ Assim como na análise do perfil dos doadores de órgãos no Brasil, no primeiro semestre de 2011, entre os 960 doadores, $36 \%$ estavam na faixa etária entre 18 e 40 anos, sendo a morte por TCE (40\% dos casos), o segundo principal motivo a $\mathrm{ME}$, ficando atrás apenas do Acidente Vascular Encefálico (48\%). ${ }^{6}$ Além disso, uma pesquisa com a população de Campinas encontrou que o não conhecimento sobre o tema doação de órgãos reduziu a opção por doar em aproximadamente três vezes. ${ }^{11}$ Dessa forma, esses dados corroboram com a importância do ensino e conscientização dos universitários sobre este tema, pois, além da grande maioria estar dentro de uma faixa etária muito prevalente entre os potenciais doadores, eles também devem estar entre os grupos mais bem informados sobre este assunto, devido suas grandes representatividades sobre a população.

No presente estudo, $65 \%$ dos jovens manifestaram intenção de doar seus órgãos, entretanto apenas $54 \%$ deles já tinham informado a algum parente sobre esse desejo. Semelhantemente, verificou-se que $61,3 \%$ dos universitários chineses tinham intenção de doar seus órgãos após a morte. ${ }^{20}$ Por outro lado, no estudo norte-americano ${ }^{21}$ realizado com 502 estudantes universitários, foi constatado que apesar de uma posição a favor da doação de órgãos dos alunos, apenas $11 \%$ deles já declararam formalmente a intenção de ser doadores fazendo seu registro no cartão de doação de órgãos. Já entre os estudantes de medicina de Belo Horizonte, 83,5\% manifestaram a intenção de doar, porém somente $61,3 \%$ dos entrevistados comunicou a família sobre sua intenção de ser doador ou não. ${ }^{22}$ Em nossa pesquisa, quando se desconhecia a intenção do familiar de tornar-se um doador, apenas um terço dos respondentes autorizaria a doação de órgãos do familiar com diagnóstico de morte encefálica. Dessa maneira, mostra-se importante a criação de campanhas incentivando os potenciais doadores a discutir o tema e comunicar a seus familiares sobre sua intenção de ser ou não doador, uma vez que, em situações já esclarecidas previamente, a decisão do familiar a favor da doação é muito mais expressiva.

Em relação aos motivos pelos quais as pessoas não doam os órgãos após a morte, o principal fator apontado pelos estudantes, nesta pesquisa, foi o desconhecimento do tema. Ao encontro desse dado, ao ser estudado o conhecimento, atitudes e comportamento de graduandos na Turquia, concluiu-se que durante a faculdade os universitários recebem um número limitado de artigos sobre saúde, o que proporciona escassa informação sobre doação, aquisição de órgãos e transplantes. ${ }^{23}$ Ainda em outra pesquisa, ${ }^{24}$ onde se aplicou um questionário para 250 universitários da Pensilvânia sobre doação de órgãos, foi constatado que o nível de conhecimento deles era deficiente e que um ensino adicional sobre esse assunto seria necessário. Entre os estudantes de medicina da USP, 89\% manifestaram a intenção de ser doador após a morte e os principais motivos para ser não doador foram por simplesmente não quererem doar $(44,1 \%)$, medo $(23,5 \%)$ e falta de informação sobre o assunto $(11,8 \%) .{ }^{8}$ Por outro lado, uma pesquisa realizada com os alunos que participaram de um programa Educacional de Doação de Órgãos e Transplantes ${ }^{25}$ demonstrou uma grande contribuição ao conhecimento e atitudes dos estudantes em relação à doação e transplante de órgãos, comprovando que projetos semelhantes a esse beneficiariam a formação de profissionais da saúde com um entendimento mais profundo sobre o processo doação-transplante.

Em uma pesquisa realizada com estudantes de Medicina, concluiuse que a maioria dos universitários pertencentes à amostra estudada tinha conhecimento sobre morte encefálica. Evidenciou-se que no decorrer do curso os estudantes foram adquirindo mais conhecimento sobre o tema, aumentando a intenção de doar. ${ }^{26}$ Dessa forma, pôdese demonstrar o quanto a educação é fundamental para melhorar as taxas de doação de órgãos, percebendo-se a grande necessidade de inclusão de matérias ou cursos específicos de doação de órgãos e tecidos para transplantes não só no currículo de todas as faculdades de Medicina, ${ }^{26}$ mas também como tópicos introdutivos nos demais cursos universitários, a fim de disseminar a informação sobre este tema a todos os universitários. Ademais, a literatura também sugere que a vontade de doar órgãos dos estudantes interfere positivamente na decisão dos seus familiares. ${ }^{22,27}$

Quando o termo "morte" foi substituído pela expressão "morte encefálica" a autorização para doação de órgãos caiu 33\%. Em paralelo, em um estudo brasileiro de base populacional, ${ }^{12}$ também ocorreu decréscimo de $20 \%$ na taxa de doação ao ser utilizada a expressão "morte encefálica". Isso sugere uma possível falta de esclarecimento sobre este termo ou uma resistência na aceitação do termo "morte encefálica" como significado de morte, inclusive no meio universitário. Da mesma forma, em outra pesquisa ${ }^{28}$ indicou o mau entendimento sobre morte encefálica como o principal motivo para as famílias negarem o consentimento à doação de órgãos.

\section{CONCLUSÃO}

A aceitação do transplante pela sociedade e a intenção de doar órgãos mostram-se absolutamente dependentes da confiança no processo, o qual se inicia no diagnóstico de morte encefálica. $\mathrm{O}$ entendimento do termo morte encefálica como sinônimo de morte demonstra-se imprescindível para a aceitação da doação de órgãos. ${ }^{12}$

Os resultados divulgados pelo $\mathrm{RBT}^{6}$ evidenciam que o número de doadores está crescendo, provavelmente, influenciado pelo número de campanhas de conscientização promovidas pela ABTO e, também, outros meios de comunicação, como revistas, jornais, rádios e televisão. Entretanto, infelizmente, o número de doadores ainda se encontra muito aquém do necessário para suprir a demanda de pacientes.

Dessa forma, percebe-se que, além da continuação dessas campanhas a favor de doação de órgãos, medidas adicionais são necessárias para atingir as metas estipuladas. Baseado na literatura, ${ }^{10,22,26}$ julga-se indispensável a inclusão de conteúdos sobre esse tema nas faculdades da área da saúde. Assim como, de acordo 
com a presente pesquisa, também se acredita que todos os cursos universitários devam abranger na sua formação uma abordagem sobre os conceitos básicos a respeito de morte encefálica e doação de órgãos, a fim de que todos os graduados tenham maior entendimento e aceitação a respeito desse assunto e, consequentemente, tornem-se um exemplo a ser seguido pela população.

\section{AGRADECIMENTOS}

Ao Programa de Educação Tutorial da Escola Superior de Educação Física da UFPel pelo auxilio fornecido através de material para impressão de questionários. À Gráfica da Universidade Federal de Pelotas pelo auxílio na impressão de questionários.

\section{ABSTRACT}

Purpose: identifying prevalence of organ donation intention in a sample among college students defining the main reasons to refuse the organ donation and verifying their understanding and acceptance as to brain death. Methods: A cross-over and randomized study. The sample included 485 freshmen. Independent variables analyzed were sex, age, type of high school, place of residence and socioeconomic level. The students' opinion on organ donation was analyzed by using direct questions about their organ donation intention, their reasons for not being donors and two similar questions, except by the replacement of the term death to brain death. Result: Of all individuals interviewed, $42.3 \%$ had age $<20$ years. As to organ donation $65.1 \%$ answered they had intention to be organ donors after their deaths. However $40.2 \%$ of students did not informed having some close relative willing to donate their organs. When asked why people do not donate their organs, "lack of knowledge" was the main reason for people's unwillingness for the organ donation after death. Most respondents (87.1\%) would authorize donation of a relative's organs after death who had previously declared their willingness to do so. However, only $60.3 \%$ would authorize it when the term "brain death" was used as death. Besides, if the interviewed person did not know their relative's opinion on organ donation, just 34\% would authorize donation. Conclusion: When the term brain death was used instead of death, the organ donation intention of students decreased $26 \%$ showing that part of them does not understand brain death as death. The understanding about this issue is necessary for the organ transplant can become a feasible therapeutic option.

Keywords: Organ Donation, Transplant, Organ Transplant, Organ Donors, Students, Knowledge, Attitude

\section{REFERÊNCIAS}

1. Coelho JC, Parolin MB, Baretta GA, Pimentel SK, de Freitas AC, Colman D. [Donor quality of life after living donor liver transplantation]. Arq Gastroenterol. 2005 Apr-Jun;42(2):83-8.

2. Lucey MR. Liver transplantation for alcoholic liver disease: past, present, and future. Liver Transpl. 2007 Feb;13(2):190-2.

3. Siminoff LA, Gordon N, Hewlett J, Arnold RM. Factors influencing families' consent for donation of solid organs for transplantation. Jama. $2001 \mathrm{Jul}$ 4;286(1):71-7.

4. Brasil: Conselho Federal de Medicina; [cited 201018 de março]; Resolução 1480]. Available from: http://www.portalmedico.org.br/resolucoes/cfm/1997/1480_1997.htm.

5. Lazar NM, Shemie S, Webster GC, Dickens BM. Bioethics for clinicians: 24 Brain death. Cmaj. 2001 Mar 20;164(6):833-6.

6. ABTO. Registro Brasileiro de TransplantesJaneiro-Junho de 2011: Available from: http://www.abto.org.br/abtov02/portugues/rbt/2011/RBTsemestral2011-parcial.pdf.

7. Amaral AS, Roza BA, Galvao FH, Jardim KM, Medina-Pestana JO. Knowledge of organ donation among one group of Brazilian professors of medicine. Transplant Proc. 2002 Mar;34(2):449-50.

8. Galvao FH, Caires RA, Azevedo-Neto RS, Mory EK, Figueira ER, Otsuzi TS, et al. [Attitude and opinion of medical students about organ donation and transplantation]. Rev Assoc Med Bras. 2007 Sep-Oct;53(5):401-6.

9. Moraes MWd, Gallani MCBJ, Meneghin P. Crenças que influenciam adolescentes na doação de órgãos. Revista da Escola de Enfermagem da USP. 2006;40:484-92.

10. Maia BO, Amorim JS. Morte encefálica: conhecimento de acadêmicos de enfermagem e medicina. JBT J Bras Transpl. 2009;2:1088-91.
11. Boteon; YL, Junior; LCdS, Marchiani; NCP, Azevedo; KDd, Boin; IdFSF, Pereira; MIW, et al. Principais razões para a doação de órgãos em uma amostra populacional na cidade de Campinas/SP. JBT J Bras Transpl. 2010;13:1303-7.

12. Tessmer CS, da Silva AR, Barcellos FC, Araujo CL, da Costa JD, Bohlke M. Do people accept brain death as death? A study in Brazil. Prog Transplant. 2007 Mar;17(1):63-7.

13. Barcellos FC. Intenção de doar órgãos em uma população adulta [tese]. Pelotas Universidade Federal de Pelotas - Anais; 2003. p. 41-2.

14. Mekeel KL, Langham MR, Jr., Gonzalez-Peralta RP, Hemming AW. Liver transplantation in very small infants. Pediatr Transplant. 2007 Feb;11(1):66-72.

15. Senouci K, Guerrini P, Diene E, Atinault A, Claquin J, Bonnet F, et al. A survey on patients admitted in severe coma: implications for brain death identification and organ donation. Intensive Care Med. 2004 Jan;30(1):38-44.

16. Lago PM, Piva J, Garcia PC, Troster E, Bousso A, Sarno MO, et al. Brain death: medical management in seven Brazilian pediatric intensive care units. J Pediatr (Rio J). 2007 Mar-Apr;83(2):133-40.

17. Zawistowski CA, DeVita MA. A descriptive study of children dying in the pediatric intensive care unit after withdrawal of life-sustaining treatment. Pediatr Crit Care Med. 2004 May;5(3):216-23.

18. Knihs; NdS, Roza; BdA, Schirmer J. Estratégias de cuidados à família no momento da perda, morte encefálica e doação de órgãos, elaboradas a partir de uma revisão de literatura de fatores que levam a família à recusa para a doação. JBT J Bras Transpl. 2010;13:1320-3.

19. Rodrigues; TK, Júnior AA. Estimativa do número de possíveis doadores de órgâos 
no estado de Sergipe no ano de 2007. JBT J Bras Transpl. 2010;13:1353-9.

20. Chen JX, Zhang TM, Lim FL, Wu HC, Lei TF, Yeong PK, et al. Current knowledge and attitudes about organ donation and transplantation among Chinese university students. Transplant Proc. 2006 Nov;38(9):2761-5.

21. Feeley TH, Servoss TJ. Examining college students' intentions to become organ donors. J Health Commun. 2005 Apr-May;10(3):237-49.

22. Júnior REO, Saldanha BO, Oliveira APP, Santos EA, Oliveira MP, Pereira WA, et al. Conhecimento sobre morte encefálica e doação de órgão entre estudantes de medicina de Belo Horizonte. JBT J Bras Transpl. 2009;12:1149-53.

23. Akgun S, Tokalak I, Erdal R. Attitudes and behavior related to organ donation and transplantation: a survey of university students. Transplant Proc. 2002 Sep;34(6):2009-11.
24. Boland KP, Baker KQ, Nicholson AB. College students perceptions about organ donation. The Journal of Heart and Lung Transplantation. 2005;24(2, Supplement 1):S133-S.

25. Garcia CD, Goldani JC, Neumann J, Chem R, Chem E, Camargo JJ, et al. Importância do programa educacional de doação e transplante em escolas médicas. JBT J Bras Transpl. 2009;12:1049-51.

26. Batista; CR, Kusterer LEFL. Conhecimento de estudantes de medicina sobre doação e transplantes de órgãos. JBT J Bras Transpl. 2010;13:1309-13.

27. Garcia CD, Barboza AP, Goldani JC, Neumann J, Chem R, Camargo J, et al. Educational program of organ donation and transplantation at medical school. Transplant Proc. 2008;40(4):1068-9.

28. West R, Burr G. Why families deny consent to organ donation. Aust Crit Care. 2002 Feb;15(1):27-32. 